\title{
Network Coding Multicast in Satellite Networks
}

\author{
Fausto Vieira ${ }^{\dagger}$ and João Barros ${ }^{\dagger}$ \\ ${ }^{\dagger}$ Instituto de Telecomunicações \\ Faculdade de Engenharia da Universidade do Porto \\ Porto, Portugal
}

\begin{abstract}
The broadcast nature of satellite communications provides a highly effective medium for content distribution, especially in the case of geographically scattered clients. In satellite networks such as DVB-RCS based systems, new approaches and further improvements in content distribution are possible. Non-real-time applications typically employ reliable multicast methods, based on application level protocols. These often make use of forward error correction (FEC) techniques and apply carousel data cycling schemes in unidirectional links. Recent developments in information theory paved the way for a groundbreaking approach for reliable multicast over satellite networks: network coding protocols that offer a native and transparent solution for reliable multicast. When employing a feedback channel, network coding can reach near real-time performance at an efficiency level close to known theoretical bounds.
\end{abstract}

\section{INTRODUCTION}

Content distribution has been a key satellite-based service for many years due to the inherent broadcast nature of satellite communications. Terrestrial-based alternatives have recently gained traction, mostly due to the ever decreasing costs of broadband communications, associated with disruptive technologies such as peer-to-peer content distribution. Nonetheless, satellite-based platforms present many important advantages that are difficult to match in a straightforward fashion. First of all, the scalability is guaranteed due to the broadcast nature of satellite communications, even when considering multi-beam satellite systems. Second, terrestrial infrastructure independence is provided by the satellite coverage ubiquity. Third, typical satellite networks present a star topology that simplifies multicast services since there is no routing. Finally, guaranteed bandwidth is usually inherent to most satellite systems, which makes it fundamental in creating a content distribution platform.

Disruptive technologies are often at the heart of novel content distribution concerns and solutions. In the context of communication networks and protocols, network coding [1], [2], offers an arguably intriguing networking concept: data throughput and network robustness can be considerably improved by allowing the intermediate nodes in a network to mix different data flows through algebraic combinations of multiple datagrams. This key idea, which clearly breaks with the standard store-and-forward paradigm of current routing solutions, is particularly valid for satellite networks where erasure patterns in large terminal populations can be countered by broadcasting encoded packets to multiple nodes simultaneously until the destination nodes have enough degrees of freedom to decode and recover the original data.

Our main contributions are two-fold: (1) efficiency improvements in reliable multicast and (2) very low decoding delay. The first originates from the lack of Forward Error Correction (FEC) overhead, which eliminates redundant data. Moreover, channel diversity minimizes redundancy in the recovery data. When comparing with fountain codes [3], a very low decoding delay is possible due to closed-loop network coding scheduling algorithms.

The impact of this approach is also two-fold: first, it provides a transparent solution for reliable multicast, which means that specific transport or application level protocols are no longer required; second, it allows for erasure-free streaming multicast, even with a long transmission path delay that is characteristic in satellite networks. The decoding delay is shown to be of the same order of magnitude as the buffering delay employed by Internet streaming applications.

The remainder of the paper is organized as follows. Related work will be covered in Sec. II before presenting our network coding multicast for satellite networks. In Sec. IV, the architectural aspects will be analyzed both at system level as well as protocol stack level. Finally, the conclusions can be found in Sec. V.

\section{RELATED WORK}

There has been a lot of research in the area of content distribution multicast, both for terrestrial and satellite networks. There is also an active IEFT Working Group, the Reliable Multicast Transport (RMT) [4] group, who recognize the need to support two reliable multicast families, namely Negative ACKnowledgement (NACK) based protocols and Forward Error Correction (FEC) asynchronous layered coding based protocols. The first family of protocols are based on the principle that receivers should only provide negative acknowledgments, thus only informing the multicast source of reception errors or losses. The latter family is based on FEC techniques based on a layered coding approach, where receivers can subscribe to multiple data streams that are transformed in such a way that reconstruction of a data object does not depend on the reception of specific data packets, but only on the number of different packets received [5].

\section{A. Reliable multicast for satellite systems}

Reliable multicast over a satellite platforms presents itself as a unique and challenging scenario, because the network topology is not an issue but rather the number of receivers 
and their different channel conditions. Furthermore, satellite systems can be unidirectional or bidirectional, where the latter allows for a high latency feedback channel. The survey in [6] analyzes qualitatively which solutions are better suited for satellite systems that can be described as a flat infrastructure. Under this scenario, the authors argue that FEC is a very good approach since it can simultaneously repair a variety of packet loss patterns. Furthermore, it is also suited for unidirectional or highly asymmetrical links [7]. The main disadvantages are related to the increased overhead and the decoding delay. In terms of satellite networks, there is a consensus that hybrid protocols present the best approach for this scenario, especially in highly asymmetrical links. However, the FEC mechanisms of hybrid protocols imply increased overhead and decoding delay, which reduces performance and can limit the scope of services and applications that are possible with reliable multicast. With Network Coding, it is possible to restrict the FEC overhead. This shall be addressed in the following section.

\section{B. Reliable Multicast for mobile satellite terminals}

The DVB-SH standard was designed for handheld terminals where a return link may not be present, thus FEC-based approaches are employed in order to cope with mobility. Nonetheless, this standard allows for the use terrestrial gapfillers and local retransmitters, thus creating path diversity.

In [8], the authors present an integrated satellite architecture based on the DVB-S2/RCS standards [9], [10] with mobility support. The proposed architecture introduces two subsystems to support unicast and Datacast services. Both subsystems are based on a FEC-only approach, even though a feedback channel is present.

Both scenarios can benefit from Network Coding techniques, since there is path diversity in the first one and a feedback channel in the second one. This will be addressed in the following section.

\section{Network Coding Multicast in SAtellite NETWORKS}

Network coding has been shown to be especially useful over wireless networks, in areas such as mobility, multipath, security and multicasting. Since satellite systems have very specific characteristics, we will present how network coding is employed in wireless networks and explain later how it is applied to satellite specific scenarios. These shall define the basis of the framework for network coding in satellite systems.

\section{A. Network Coding in wireless networks}

The broadcast nature of wireless networks provides an ideal medium for network coding. In the relay network of Fig. 1 with one relay (R) and two end nodes (A,B), four transmissions are usually necessary in order to exchange two messages between the end nodes. With network coding, it is possible to reduce to three transmissions because the relay transmits a linear combination of the symbols that form the messages. Each end node is aware of its own message and corresponding symbols and therefore is able to decode the transmission sent by the relay node and extract the incoming message.

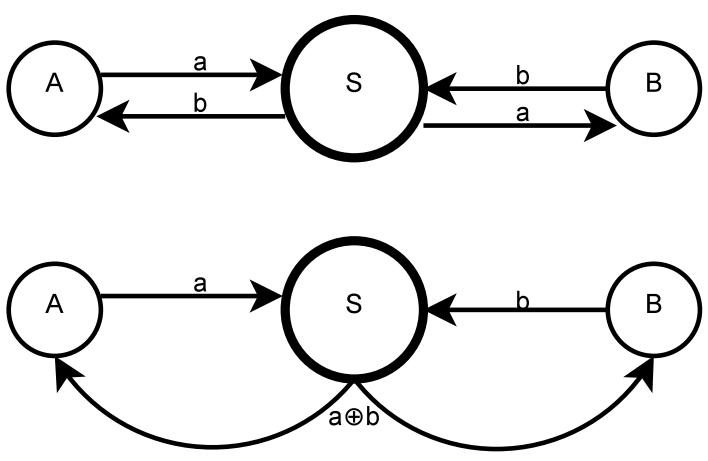

Fig. 1. Tradicional routing versus network coding

In wireless networks, it is also common to have multipath connections due to the broadcast nature of the network. Only one path is selected with routing protocols, thus limiting the capacity to the achievable capacity in that path. With network coding, it is possible to reach the min-cut capacity by transmitting linear encoded symbols through the different paths. The messages are decoded when enough linear independent messages have been received. This corresponds to the mincut capacity, i.e. to the maximum flow between the two nodes when all possible paths are used.

Multicasting over wireless networks can also be greatly improved with network coding. Messages are linearly encoded so that receivers can decode them after enough independent symbols have been received. This allows receivers to mitigate erasures especially when a feedback channel is present. Moreover, linearly encoded symbols can be optimized simultaneously for all receivers according to the actual erasure patterns

\section{B. Netcork Coding in Satellite Networks}

We now consider a content distribution multicast scenario for typical satellite network topologies. There are many different return channel technologies in satellite networks, although the return channel over satellite is a common reference scenario. These networks are usually characterized by having highly asymmetrical links with long round-trip delays.

In terms fixed satellite terminals, the erasure rates are relatively small, although slow fading events caused by weather conditions can affect groups of terminals within the same geographical region. the erasure rates can differ from the unidirectional satellite systems if the Fade Mitigation Techniques (FMT) are employed. These can be used to adapt signal protection levels, which affects the erasure rates as well as available capacity. It is foreseen that multicast services employ population management techniques in order to guarantee low erasures rates for most receivers. This is only possible with FMT techniques. Either with fixed or variable capacity systems, network coding is especially useful since it can provide time-diversity for non-real-time services by combining previously transmitted symbols with new ones. Furthermore, it can use the feedback 
channel to optimize the linear combinations according to the specific erasures reported by the receivers. The performance gains and scalability features are shown in section IV. When employing multicast population management techniques, it is possible to optimize performance since network coding reduces the need for very high protection levels at the physical layer, thus increasing the available capacity.

In terms of mobile satellite terminals, these are both affected by the slow fading events present in fixed satellite terminals as well as fast fading events that are characteristic of mobility related impairments. Network coding can provide short scale time diversity, which allows the receivers to overcome the erasure patterns caused by fast fading events. However, the feedback channel can also be used to optimize the linear combinations according to the specific erasures reported by the terminals. Due to the fast fading events that are typical of mobile satellite terminals, only FEC-based approaches were possible with traditional approaches. Therefore, network coding approaches can present great performance gains with the reduction or elimination of the FEC related overhead. Note that it is possible to introduce some network coding redundancy similar to FEC, in order to reduce erasures and limit the use of the feedback channel. This may be recommended for networks where the return link resources are scarce, which is quite common for satellite networks. Some mobile satellite systems employ terrestrial gap-fillers that enhance the satellite coverage, as shown in Fig. 2. They can also provide local content that is not carried over satellite. Multipath capabilities are also foreseen for satellite networks such as in the railway scenario, where tunnels, train stations and urban environments produce channel impairments for long time periods. In this scenario, network coding can represent significant improvements by taking advantage of the multipath capabilities. Independent linear combinations transmitted through multiple paths allows for receivers to decode the messages as soon as it has received enough encoded symbols. This means that multiple paths not only provide redundancy but also enhanced performance by achieving the min-cut capacity.

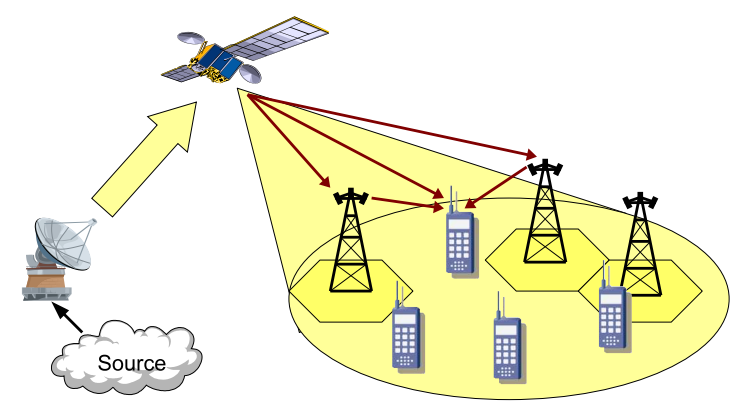

Fig. 2. Simulator block diagram

\section{Architectural Aspects}

This section focuses on the broad scenario content distribution over satellite networks with fixed and mobile terminals.
This means slow fading events affect every terminal and that fast fading events affect the mobile terminals. Furthermore, every terminal can use the return link to inform the source of the erasure losses, in order to take full advantage of network coding benefits.

\section{A. System Model}

The system model is based on a typical flat infrastructure in a star topology. Basically, any broadband satellite system with a return channel falls within the scope of the system model in Fig. 3. It is generic enough to accomodate satellite systems

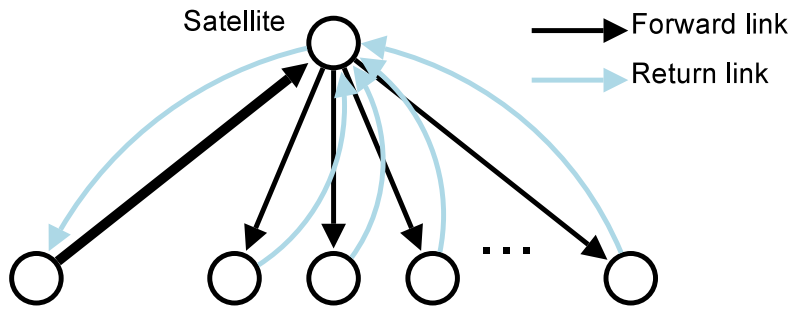

HUB

Fig. 3. Simulator block diagram

with FMT techniques, multi-beam satellites, as well as fixed and mobile terminals, provided that every terminal is able to transmit feedback information.

\section{B. Protocols and algorithms}

The core of the network coding multicast lies on the scheduling and error recovery algorithms. These must take advantage of the feedback channel in order to maximize performance and minimize decoding delay. Furthermore, the encoding symbols must be carried over network coding protocols.

1) Protocols: Satellite systems usually employ encapsulation protocols to carry network datagrams, namely IP packets. The requirements for an encapsulation protocol to support network coding are quite straightforward: sequence or generation number, coeficients matrix and payload. Using the same methodology as in [8] for the FEC support over Multiple Protocol Encapsulation (MPE), it possible to redefine certain header fields that are not used in datacast applications. In the case of Generic Stream Encapsulation (GSE), the extension header mechanisms that are located in this protocol allow for the introduction of new features such as network coding support. The use of existing encapsulation protocols to support network coding allows for simplified deployment. Furthermore, the location of these protocols just below the network layer in the protocol stack allows for network coding to be transparent to the higher layers including the network layer.

2) Network Coding Algorithms: The network coding algorithms is the key element for obtaining the promised performance gains. We now oulline some of the fundamental requirements when designing these algorithms for the case of satellite networks. 
First of all, the transmission should have a very low decoding delay, which is equivalent to the intermediate performance of Fountain codes. This low decoding delay is required for near real-time services even though it carries a small loss of efficiency. From [11], it is shown that for fountain codes, the intermediate performance for a small number of received coded symbols is obtained with a degree of $d=1$. In terms of Network Coding, this means that most symbols should be transmitted as is, i.e. without combining them with other symbols.

The second requirement is that symbols that were already seen should not be transmitted again. This can be be achieved by employing a sliding window mechanism [12]. The scheduler will only transmit symbols within this sliding window.

The last requirement is that the error recovery process should employ linear combinations in such a way that every symbol can be fully recovered with a minimum number of retransmitted symbols.

\section{Performance gains}

The performance gains are focused in comparing the previously presented scenario to the traditional NACK-based reliable multicast, which means that a symbol retransmission occurs until every receiver successfully receives the symbol. Traditional NACK-based approaches present serious scalabil-

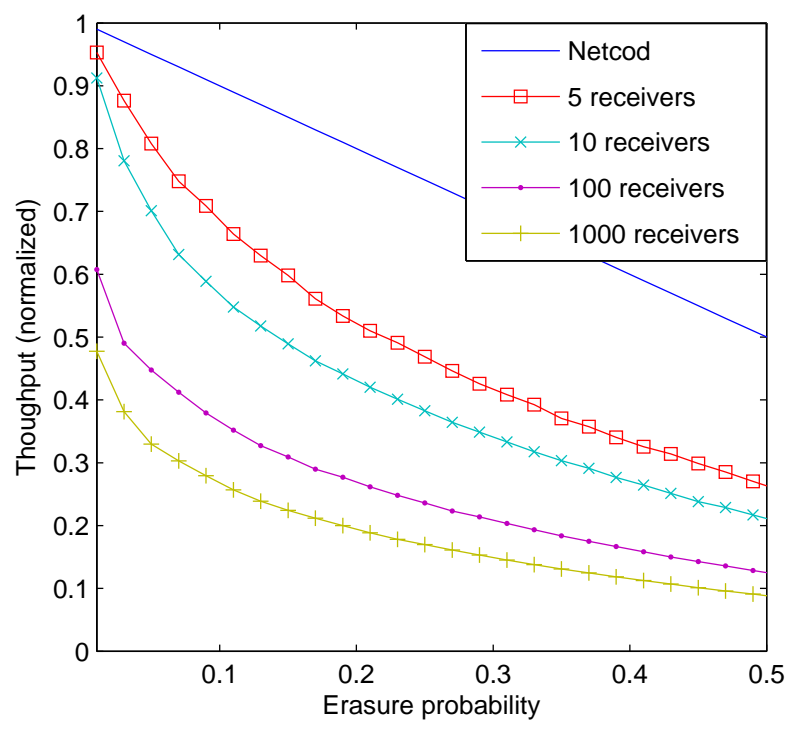

Fig. 4. Network coding multicast only requires each node to receive enough symbols in order to decode the data. On the other hand, NACK-based multicast requires that each node receives every symbol in order to decode the data.

ity issues, as shown in Fig. 4. Satellite systems can cover very wide geographical areas with large number of terminals and therefore scalability is a fundamental performance indicator for these systems.

\section{CONCLUSiON}

The presented framework for transparent multicast based on network coding approaches was shown to be applicable to both unidirectional and bidirectional satellite systems, as well as fixed and mobile terminals. We also addressed the multipath scenario that is possible in DVB-SH systems. Furthermore, the simulation results show great improvements in terms of performance between traditional and network coding approaches. This performance is more relevant for scenarios with large number of terminals, which is the case for most satellite systems.

We demonstrated that network coding can take advantage of satellite systems' inherent charactistics in order to push performance beyond the limits present with traditional approaches and close to the theoretical bounds.

\section{REFERENCES}

[1] R. Ahlswede, N. Cai, S.-Y. Li, and R. Yeung, "Network information flow," Information Theory, IEEE Transactions on, vol. 46, no. 4, pp. 1204-1216, Jul 2000.

[2] P. A. Chou, Y. Wu, and K. Jain, "Practical network coding," in 41st Allerton Conf. Communication, Control and Computing, Monticello, IL, US, Oct 2003.

[3] A. Shokrollahi, "Raptor codes," Information Theory, IEEE Transactions on, vol. 52, no. 6, pp. 2551-2567, June 2006.

[4] http://www.ietf.org/html.charters/rmt-charter.html.

[5] http://www.ietf.org/internet-drafts/draft-ietf-rmt-bb-lct-revised-07.txt.

[6] M. W. Koyabe and G. Fairhurst, "Reliable multicast via satellite: a comparison survey and taxonomy," International Journal of Satellite Communications, vol. 19, no. 1, pp. 3-28, 2001.

[7] P. Basu and K. Kanchanasut, "A reliable multicast protocol for unidirectional satellite link," in SAINT '03: Proceedings of the 2003 Symposium on Applications and the Internet. Washington, DC, USA: IEEE Computer Society, 2003, p. 390.

[8] F. Vieira, M. A. Vázquez Castro, and J. Lei, "Datacast Transmission Architecture for DVB-S2 Systems in Railway Scenarios," in SPCS '08: 10th International Workshop on Signal Processing for Space Communications, Rhodes Island, Greece, October 2008.

[9] European Telecommunications Standards Institute, EN 302 307: Digital Video Broadcasting (DVB): Second generation framing structure, channel coding and modulation systems for Broadcasting, Interactive Services, News Gathering and other broadband satellite applications. Sophia Antipolis, France: ETSI, June 2006.

[10] Digital Video Broadcasting, Interaction channel for Satellite Distribution Systems (draft EN 301790 V1.5.1 - DVB-RCS+M). Geneva, Switzerland: DVB, July 2008.

[11] S. Sanghavi, "Intermediate performance of rateless codes," Information Theory Workshop, 2007. ITW '07. IEEE, pp. 478-482, Sept. 2007.

[12] J. K. Sundararajan, D. Shah, and M. Médard, "ARQ for network coding," in Proceedings of the IEEE International Symposium on Information Theory (ISIT 2008), Toronto, Canada, July 2008. 